\title{
Microwave Tomographic Imaging Utilizing Low-Profile, Rotating, Right Angle-Bent Monopole Antennas
}

\author{
N. R. Epstein, ${ }^{1}$ P. M. Meaney, ${ }^{2}$ and K. D. Paulsen ${ }^{2,3,4,5}$ \\ ${ }^{1}$ Schulich School of Engineering, University of Calgary, 2500 University Drive NW, Calgary, AB, Canada T2N 1N4 \\ ${ }^{2}$ Thayer School of Engineering, Dartmouth College, 14 Engineering Drive, Hanover, NH 03755, USA \\ ${ }^{3}$ Department of Radiology, Geisel School of Medicine, Dartmouth College, Hanover, NH 03755, USA \\ ${ }^{4}$ Norris Cotton Cancer Center, Dartmouth Hitchcock Medical Center, Lebanon, NH 03756, USA \\ ${ }^{5}$ Advanced Surgical Center, Dartmouth Hitchcock Medical Center, Lebanon, NH 03756, USA
}

Correspondence should be addressed to N. R. Epstein; nepstein@ucalgary.ca

Received 2 May 2014; Accepted 5 June 2014; Published 29 June 2014

Academic Editor: Dau-Chyrh Chang

Copyright (c) 2014 N. R. Epstein et al. This is an open access article distributed under the Creative Commons Attribution License, which permits unrestricted use, distribution, and reproduction in any medium, provided the original work is properly cited.

\begin{abstract}
We have developed a simple mechanism incorporating feedline bends and rotary joints to enable motion of a monopole antenna within a liquid-based illumination chamber for tomographic imaging. The monopole is particularly well suited for this scenario because of its small size and simplicity. For the application presented here a full set of measurement data is collected from most illumination and receive directions utilizing only a pair of antennas configured with the rotating fixture underneath the imaging tank. Alternatively, the concept can be adapted for feed structures entering the tank from the sides to allow for measurements with vertically and horizontally polarized antennas. This opens the door for more advanced imaging applications where anisotropy could play an important role such as in bone imaging.
\end{abstract}

\section{Introduction}

Microwave tomography (MT) is a nonionizing imaging modality capable of noninvasively recovering a wide range of dielectric property (DP) values [1-3]. An observable contrast exists between the dielectric properties (e.g., permittivity and conductivity) of healthy and abnormal breast tissue [48], and MT has shown great promise as a clinical imaging technique for applications initially related to breast-cancer detection [9], diagnosis [8], and chemotherapy monitoring [10]. Current breast-imaging investigations at Dartmouth College (Hanover, NH, USA) now focus on multimodal MRMT techniques [11-14], with the advent of MT providing specificity information to the high-resolution noncontrast enhanced MR images. The development of an MR compatible MT apparatus required array profile reductions due to size restrictions associated with its placement inside the MR bore. By decreasing antenna feedline lengths within the imaging chamber, signal corruption increased due to the presence of less-heavily attenuated surface wave-based multipath signals [15]. These investigations were foundational steps in understanding signal corruption issues associated with miniaturizing monopole antenna-array elements for biomedical microwave tomography.

One of our current interests is imaging and assessing the DPs of bone. Bone mineral density (BMD), along with additional bone-quality factors such as bone architecture, is currently used as clinical indicators of bone health. These measurable characteristics have enormous clinical potential as osteoporotic-screening and ultimately bone health assessment tools [16]. Investigations evaluating bone's DP variations as a function of mineralization using excised, demarrowed, trabecular porcine-bone samples indicate a strong correlation between the MT recovered DPs and bone-volume fraction measurements acquired using micro-CT [17]. In fact, the first clinical microwave tomographic imaging of the human calcaneus has recently been reported [18], further highlighting the clinical applicability of using MT for assessing the health of bone using DP measurements. Dartmouth's current clinical imaging system, comprehensively described 


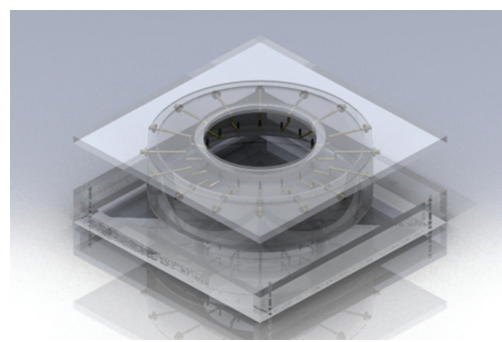

(a)

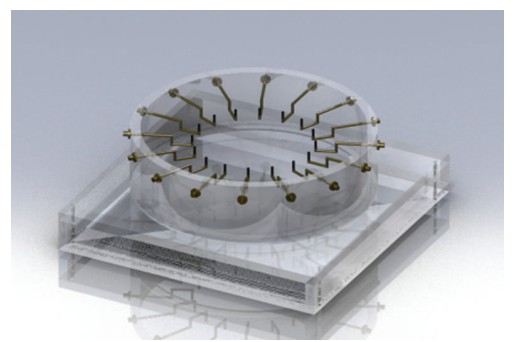

(b)

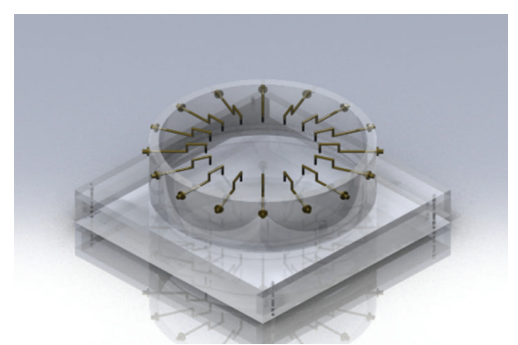

(c)

FIGURE 1: SolidWorks rendering of an example rotary-enabled monopole antenna array: (a) with top cover, (b) with antennas oriented upwards, and (c) with antennas oriented downwards.

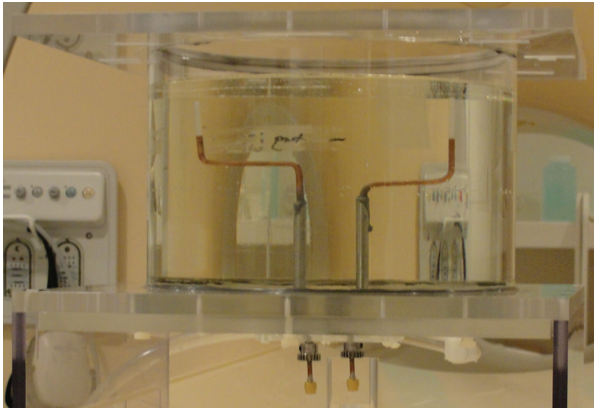

(a)

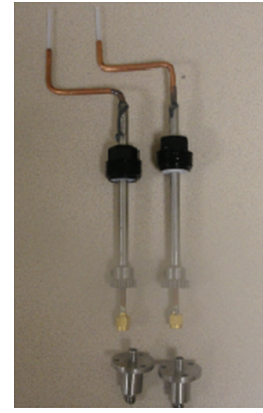

(b)

FIGURE 2: Photographs of (a) the new feed construct and antennas placed inside the imaging chamber and (b) the two bent monopoles inserted through their hydraulic seals prior to rotary joint attachment.

in [19], employs an array of 16 monopole antennas. Although free-space operation presents numerous difficulties for the monopole [20-22], operation in the system's lossy-coupling medium substantially broadens the antenna's frequency response due to resistive loading, a significant advantage in that it provides full target coverage without unwanted blind spots. Low-cost and easily constructed, the monopole's design presents many advantages in the context of our multichannel motion enabled array system, including ease of modeling [23] and positioning close to the target's surface in an array due to low mutual coupling. A thorough characterization of the system's antenna elements in terms of return loss, effective beam width, and beam steering angle as a function of electrical length can be found in [24].

The DP characteristics of bone are unique due to the complex structure of the underling bone tissue. Recent studies have demonstrated that the microstructure is highly correlated with the anisotropic DP distributions of trabecular bone [25]. Although our noncontacting antenna array design permits vertical translation of the elements, their orientations are fixed parallel to one another. In order to image these anisotropies with our system, antenna elements with the ability to transmit and receive signals in orthogonal orientations are needed. This can be accomplished clinically with the advent of antenna-array elements that can pivot around a predetermined axis using a rotary joint (Cobham, Kelvin Corporation, Rotary-Coupler Part Number 1106SS). We have chosen this approach rather than simply adding additional antennas into the array as they increase both cost and the electronics' complexity. Obtaining the additional range of polarization orientations necessarily requires the use of a novel rotary mechanism to avoid damaging feedlines from repeated twisting. Figure 1 shows CAD renderings of a proposed low-profile imaging chamber that incorporates antenna rotation utilizing right angle-bent monopole antennas in conjunction with rotary joints for feedlines entering the tank from the sides. The antenna feedline-bending pattern inside the illumination chamber ensures that the center of the antenna is at the same physical location despite the rotation of the feedline at the tank interface.

To evaluate the clinical feasibility of incorporating this new data collection strategy into a biomedical microwave imaging system, we have conducted a pilot investigation to analyze the use of rotating, rotary joint-enabled, right anglebent monopole antennas in a prototype two-antenna MT array (Figure 2). These miniaturized monopoles have reduced profiles within the imaging chamber due to the bending configuration, and the introduced rotational capability directly increases the number of independent measurement positions for a single monopole. Presented are S-parameter measurements of the straight and bent monopoles, with and without the introduction of the rotary joint. Minimal deviations are observed after including both bends and rotary joints in the antenna construct, indicating the design modifications have not degraded the antenna's performance over the system's operating frequency range $(500-3000 \mathrm{MHz})$. Our system is designed to operate within this range due to the recognized dielectric property contrast that exists between healthy and 


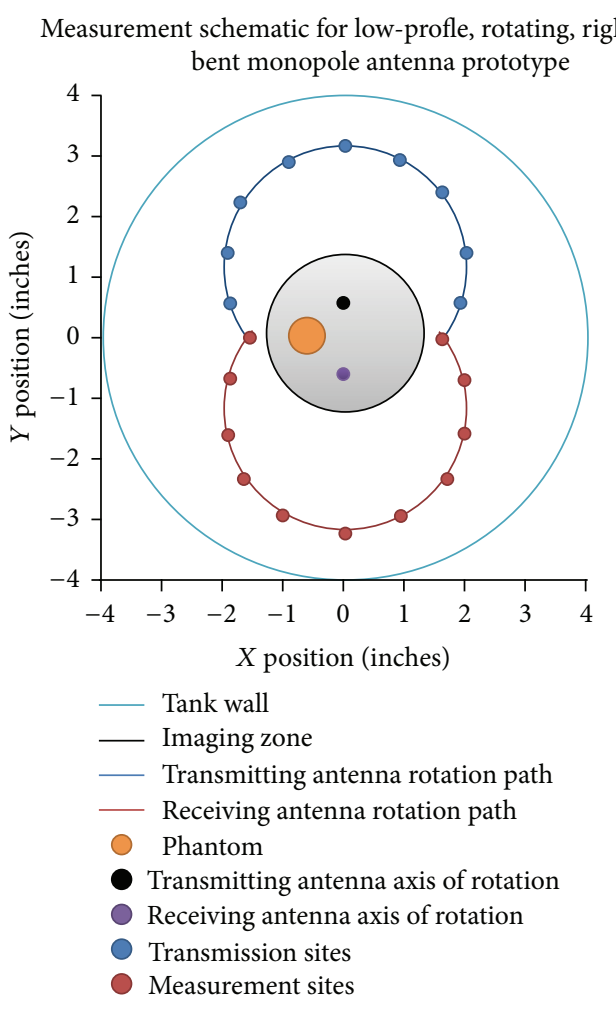

(a)

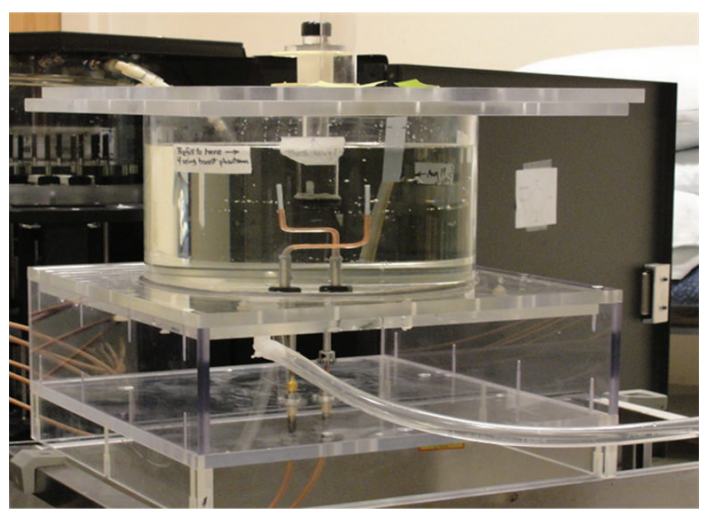

(b)

Figure 3: (a) 2D schematic of the experimental-imaging set-up and (b) side-view photograph of the imaging chamber with inclusion region during data collection.

abnormal tissues over this frequency span [4-8]. In addition, we have shown previously that it is difficult to transmit a signal across the target zone in just the homogeneous bath at frequencies above $3000 \mathrm{MHz}$ [26]. Reconstructed MT images from simulated and measured data are also shown, highlighting the profile-reduced rotary-enabled prototype's ability to recover high-caliber electric field data for accurate image reconstruction using a unique, noncircular data collection configuration.

\section{Methods}

2.1. Experimental Set-Up and Antenna Positioning. The antenna and feed structure was constructed out of $2.3 \mathrm{~mm}$ diameter semi-ridged coaxial cable with a $3.4 \mathrm{~cm}$ lengthexposed tip forming the radiator's active region. Bending of the monopole was accomplished using a ridged mold that allowed slow, progressive, and precise maneuvering of the feedline into the desired angular geometry. This decreased the monopole's profile within the imaging chamber while maintaining an appropriate feedline length for reduced multipath corruption from surface waves. The antennas enter the imaging chamber through hydraulic seals in the tank's bottom and are fixed at a specific vertical location within the tank using short stainless-steel sleeves and conductive silver epoxy. Their attachment to the rotary joints is outside of the imaging chamber. Accurate positioning of the elements to numerous signal transmission and detection positions was easily achieved by incorporating an external-gearing mechanism.

Figure 3 shows a schematic of the experimental imaging set-up. In this two-antenna prototype, antenna A (the dedicated transmitter) was physically moved to 9 transmission positions while antenna $B$ (the dedicated receiver) was moved to 11 receiving positions via the use of the rotary joint in conjunction with the modified antenna geometry. Note that because the centers of rotation for each feedline were not coincident, the shape of the imaging zone was noncircular. The collected measurements were mixed with a frequencydependent local oscillator, and downconverted, low-pass filtered intermediate frequency signals are sent to the DAQ for sampling. The field measurements collected at each transmitreceive pair were used in our offline image reconstruction algorithm, which is briefly outlined in the following section.

2.2. Data Acquisition and Image Reconstruction. Electric field amplitude and phase measurements related to the objectunder-test (OUT) were obtained by subtracting a calibration data set (with only coupling medium present in the imaging system) from the measurements taken with the OUT submerged in the lossy-medium, under the same data acquisition protocol. This process is outlined in

$$
E_{\mathrm{OUT}}^{m}=E_{\mathrm{OUT} b}^{m}-E_{b}^{m},
$$




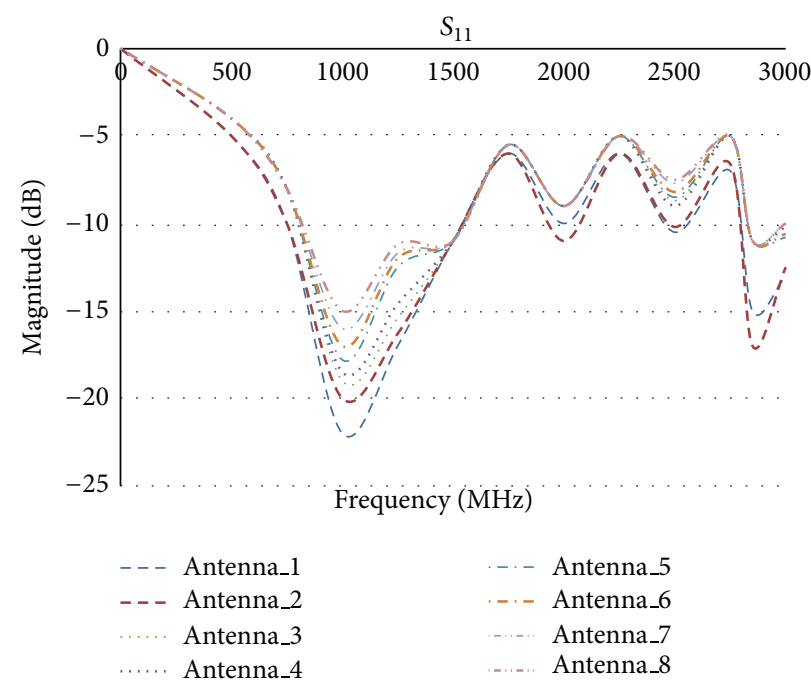

FIGURE 4: Measured return loss for (a) the clinical imaging system's traditional encapsulated monopoles (antenna_1 and antenna_2, resp.), (b) the straight monopoles prior to bending (antenna_3 and antenna_4, resp.), (c) the bent monopoles (antenna_5 and antenna_6, resp.), and (d) the bent monopoles incorporating the rotary joint (antenna_7 and antenna_8, resp.).

where $E_{\mathrm{OUT}}^{m}$ are the measured field values of the OUT, $E_{\mathrm{OUT} b}^{m}$ are the combined measured field values of the OUT and background coupling-medium, and $E_{b}^{m}$ are the measured field values of the background coupling-medium, respectively. Subtractions were all calculated in log magnitude and phase format.

Microwave image reconstruction requires solving an illposed, nonlinear inverse-scattering problem with an iterative computational method. Using a dual mesh approach [27], we have implemented a Gauss-Newton iterative technique with a log-transform [28] and Tikhonov regularization $(\lambda)$ [29] to solve the wave equation for the squared complex-value wave number $\left(k^{2}\right)$. The forward solution for each iteration is calculated using a hybrid-element method described in [30]. The algorithm seeks to minimize the squared error objective function for the measured and calculated amplitude and phase values. The log magnitude/phase formulation of this function is represented by

$$
\begin{aligned}
\Omega= & \left\|\Gamma^{m}-\Gamma^{c}\left(k^{2}\right)\right\|_{2}^{2} \\
& +\left\|\Phi^{m}-\Phi^{c}\left(k^{2}\right)\right\|_{2}^{2} \\
& +\lambda\left\|L\left(k^{2}-k_{o}^{2}\right)\right\|_{2}^{2},
\end{aligned}
$$

where $\Gamma^{m}, \Gamma^{c}, \Phi^{m}$, and $\Phi^{c}$ are the log magnitude and phase values for the measured and computed field values, respectively. $L$ is a regularization matrix and $k_{o}^{2}$ is a prior estimate of $k^{2}$. Simulated data corresponding to the actual measurement set-up was generated using a finite element forward solver described in [29] and includes the addition of simulated noise levels ranging from -80 to $-100 \mathrm{dBm}$.

The algorithm uses the dielectric property distribution of the background emersion medium as an initial estimate. The emersion medium's DPs can vary up to $3 \%$ as a function of nominal room temperature fluctuations. Internal experiments have shown that our imaging algorithm is robust and produces only minor artifacts even when the assumed bath properties differ from the actual one's by as much as $15 \%$. In this context, temperature variations have minimal impact on overall image quality.

\section{Results}

3.1. Return Loss Measurements. To analyze the effect of introducing a right angle bend and rotary joint into the monopole antenna-feed network, $S_{11}$ measurements were collected (in an 80:20 glycerine: water mixture) using an Agilent E5071A network analyzer for (a) the clinical imaging system's traditional encapsulated monopoles (antenna_1 and antenna_2, resp.), (b) the straight monopoles prior to bending (antenna_3 and antenna_4, resp.), (c) the bent monopoles (antenna_5 and antenna_6, resp.), and (d) the bent monopoles incorporating the rotary joint (antenna_7 and antenna_8, resp.) (Figure 4).

Comparing the return loss for our clinical monopoles (i.e., antenna_1 and antenna_2,) to those of the straight, bent, and rotary joint enabled monopoles demonstrates negligible performance changes, indicating that the use of the right angle-bent monopole in conjunction with the rotary joint is acceptable for this miniaturized microwave-imaging investigation. Even with the addition of the bend and rotary joint, the antenna's resonant frequency remained roughly at $1000 \mathrm{MHz}$, and its characteristic performance was consistent for all design modifications.

In general, our monopole antennas have return losses on the order of -8 to $-9 \mathrm{~dB}$ out at the frequencies extremes [24]. In the worst case, $-5 \mathrm{~dB}$ of transmitted power is reflected (32\%). This implies that $68 \%$ is transmitted $(-1.7 \mathrm{~dB})$. While not optimal, given that our measurement system can detect signals down to $-140 \mathrm{dBm}$ and our typical transmission 

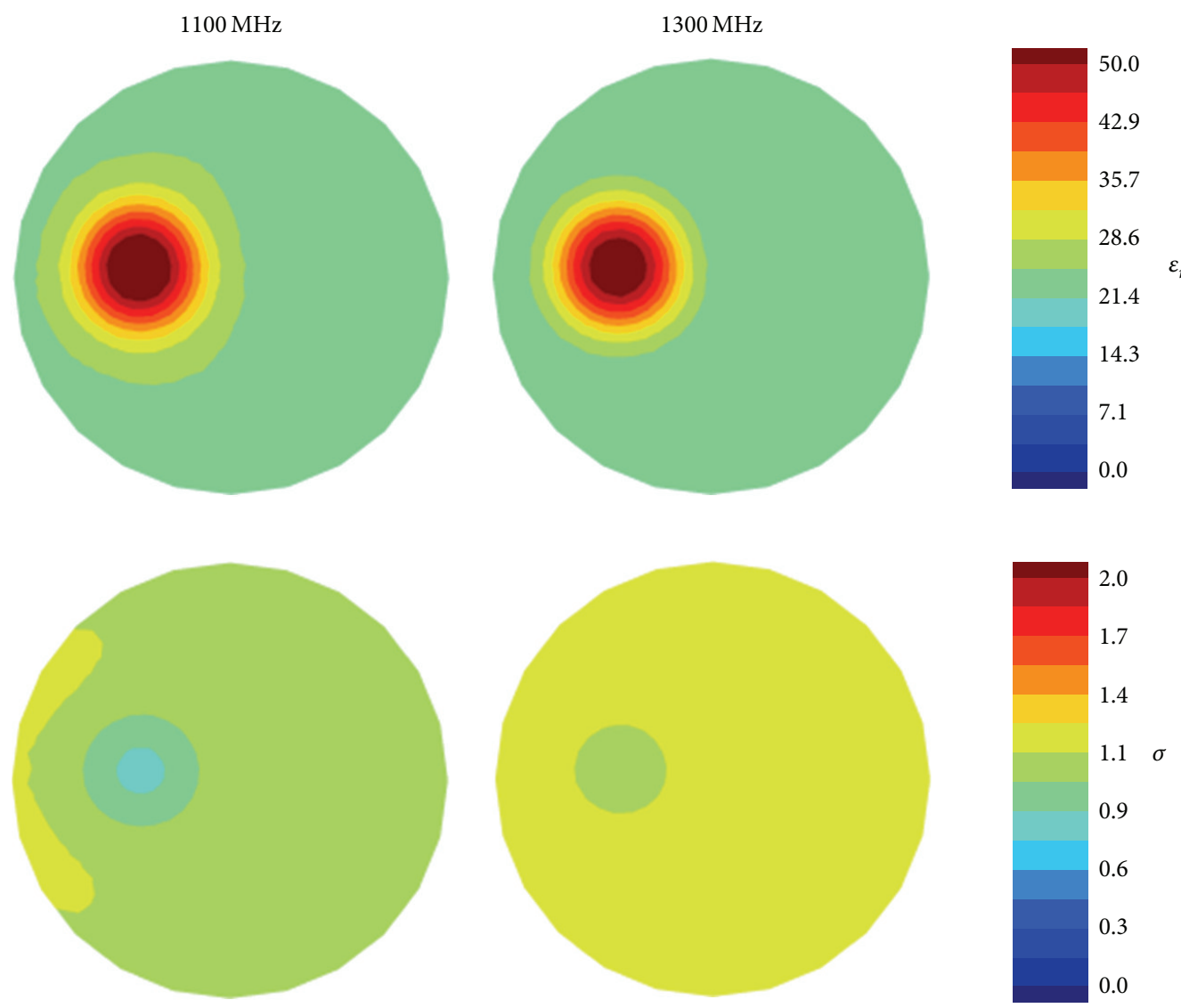

FIGURE 5: Reconstructed 1100 and $1300 \mathrm{MHz}$ permittivity and conductivity images from simulated data.

power is roughly $1 \mathrm{~mW}$, we still receive enough signal to maintain a healthy signal-to-noise ratio. It should also be noted that the periodic humps seen from 1500 to $3000 \mathrm{MHz}$ suggest the presence of standing waves resulting from the long feedline length between the reference plane for the network analyzer set during the calibration process and the active part of the antenna. Incorporating longer transmission lines that were in fact part of the whole feed structure increased the distance between the calibration reference plane and the actual antenna.

\subsection{Reconstructed Images from Simulated and Measured Data.} 1100 and $1300 \mathrm{MHz}$ reconstructed permittivity and conductivity image sets using simulated noisy data representative of the experimental set-up and actual measured data are shown in Figures 5 and 6, respectively. The mixture ratios and independent probe-measured dielectric properties of the background and inclusion regions, as well as the recovered DP values at the midpoint of the inclusion regions, are summarized in Tables 1 and 2, respectively. This simulation indicates that the novel rotary-enabled two-antenna array measurement set-up of 9 transmission positions and 11 measurement sites can facilitate the recovery of accurate dielectric property values for the background and the inclusion region, as well as their relative positions. The inclusion region is identifiable in all of the reconstructed images, especially for the permittivity, where the contrast between the background and inclusion
TABLE 1: Probe collected dielectric property values for the bath and inclusion region including their mixture composition (1100 and $1300 \mathrm{MHz}$ ).

\begin{tabular}{lccccc}
\hline Region & $\begin{array}{c}\text { Mixture ratio } \\
\text { (by weight) }\end{array}$ & \multicolumn{2}{c}{$\begin{array}{c}\text { Actual values } \\
(1100 \mathrm{MHz})\end{array}$} & \multicolumn{2}{c}{$\begin{array}{c}\text { Actual values } \\
(1300 \mathrm{MHz})\end{array}$} \\
\hline Bath & $\begin{array}{c}80: 20 \\
\varepsilon_{r}\end{array}$ & $\begin{array}{c}\sigma \\
{[\mathrm{S} / \mathrm{m}]}\end{array}$ & $\varepsilon_{r}$ & $\sigma[\mathrm{S} / \mathrm{m}]$ \\
\hline Inclusion & $\begin{array}{c}50: 50 \\
\text { glycerine }: \text { water } \\
\text { glycerine: water }\end{array}$ & 53.3 & 1.1 & 25.3 & 0.97 \\
\hline
\end{tabular}

region is highest. The enhanced recovery of the inclusion region observed in the $1100 \mathrm{MHz}$ conductivity image, when compared to the $1300 \mathrm{MHz}$ image, is due to the larger DP contrast that exists between the background and inclusion region at that frequency $(0.3 \mathrm{~S} / \mathrm{m}$ as compared to $0.1 \mathrm{~S} / \mathrm{m})$.

\section{Conclusion}

In this report, we studied the performance of the monopole antenna while incorporating several novel features into the feed network-namely, right angle bends near the active parts and rotary joints to allow for rotation about the primary axis of the feedline. While these constructs may be relatively commonplace in a range of microwave applications, this 

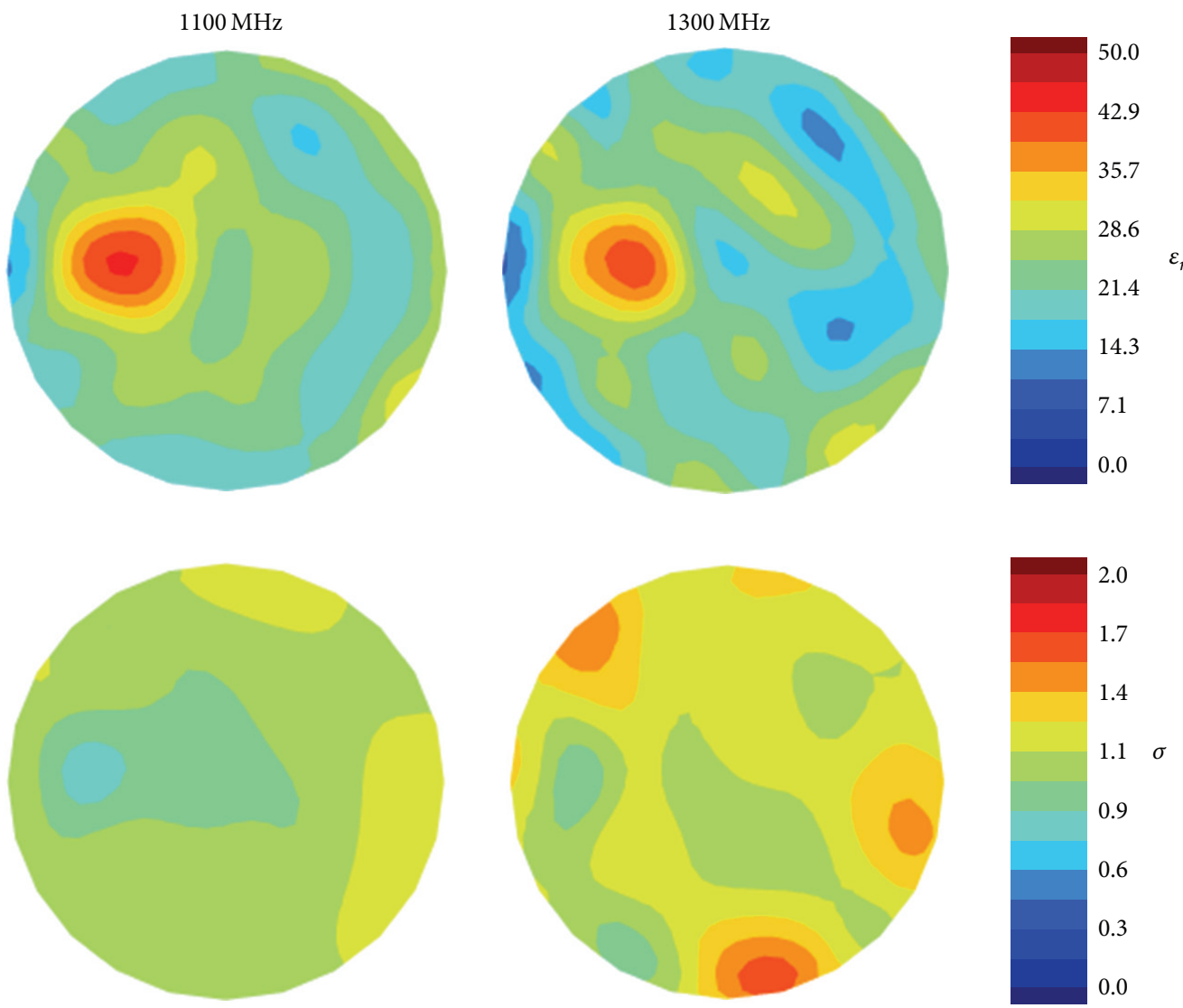

FIGURE 6: Reconstructed 1100 and $1300 \mathrm{MHz}$ permittivity and conductivity images from measured data.

TABLE 2: Recovered dielectric properties values at the midpoint of the inclusion region for the simulated and measured data (1100 and $1300 \mathrm{MHz}$ ).

\begin{tabular}{ccccc}
\hline & \multicolumn{2}{c}{$\begin{array}{c}\text { Value at midpoint of } \\
\text { inclusion region } \\
\text { (simulated data) }\end{array}$} & \multicolumn{2}{c}{$\begin{array}{c}\text { Value at midpoint of } \\
\text { inclusion region } \\
\text { (measurement data) }\end{array}$} \\
\hline$\varepsilon_{r}$ & $\sigma[\mathrm{S} / \mathrm{m}]$ & $\varepsilon_{r}$ & $\sigma[\mathrm{S} / \mathrm{m}]$ \\
\hline $1100 \mathrm{MHz}$ & 57.3 & 0.91 & 48.52 & 0.95 \\
$1300 \mathrm{MHz}$ & 54.84 & 1.23 & 42.03 & 1.04 \\
\hline
\end{tabular}

is the first report of their incorporation into biomedical MT applications, and in combination they open a range of possibilities for microwave tomographic imaging. Being able to remotely alter both antenna position and orientation instantly increases the design degrees of freedom with respect to system configuration. It is important that the antenna characteristics remained relatively constant even with these transmission-line perturbations. For the imaging results presented here, the data was no longer restricted to an array of antennas configured strictly on a circular arc. This illustrated that good quality images can be recovered in nonstandard array configurations, which opens up the possibility for further reducing the number of antennas and associated electronics channels, significantly reducing overall costs and simplifying the system layout.
More importantly, it opens up the possibility of conveniently acquiring measurement data from a range of polarization orientations-especially for feedlines entering the tank from the sides. As discussed earlier, for applications such as bone imaging, being able to reconstruct the anisotropic dielectric property distributions could pave the way towards noninvasively recovering bone structural information. This would be a significant advance in monitoring bone-health, which is an increasingly important health concern. Finally, these analyses further emphasize that the monopole antenna generally offers a range of electrical and practical advantages over a host of alternate conventional approaches.

\section{Conflict of Interests}

Drs. P. M. Meaney and K. D. Paulsen cofounded Microwave Imaging System Technologies (MIST), Inc., in Hanover, NH. MIST holds six patents, of which three are relevant to microwave breast tomography. In addition, Drs. P. M. Meaney and K. D. Paulsen are coinventors on two patents through Dartmouth College which are peripherally related to microwave breast tomography. There are additional three patents pending through Dartmouth College related to microwave breast tomography. MIST won an NIH/NCI SBIR Phase I grant in 1998 and a follow-on Phase II grant in 2002. It also collaborated with the Electronics and Telecommunications Research Institute (ETRI) in Daejeon, Republic of Korea, 
from 2007 to 2010. It is possible that Drs. P. M. Meaney and K. D. Paulsen could benefit financially from their microwave imaging patents. Dr. N. R. Epstein is a coinventor on a patent pending through Dartmouth College related to microwave imaging. It is possible that Dr. N. R. Epstein could benefit financially from this pending patent, if it is granted.

\section{Acknowledgments}

This work was conducted by Dr. N. R. Epstein while at the Thayer School of Engineering (Dartmouth College, Hanover, $\mathrm{NH}$, USA), with financial support provided by NIH/NCI Grant no. PO1-CA080139 and NSERC CREATE I3T Program.

\section{References}

[1] P. M. Meaney, M. W. Fanning, D. Li, S. P. Poplack, and K. D. Paulsen, "A clinical prototype for active microwave imaging of the breast," IEEE Transactions on Microwave Theory and Techniques, vol. 48, no. 1, pp. 1841-1853, 2000.

[2] D. Li, P. M. Meaney, T. Raynolds, S. A. Pendergrass, M. W. Fanning, and K. D. Paulsen, "Parallel-detection microwave spectroscopy system for breast imaging," Review of Scientific Instruments, vol. 75, no. 7, pp. 2305-2313, 2004.

[3] V.Zhurbenko, T. Rubæk, V. Krozer, and P. Meincke, "Design and realisation of a microwave three-dimensional imaging system with application to breast-cancer detection," IET Microwaves, Antennas and Propagation, vol. 4, no. 12, pp. 2200-2211, 2010.

[4] H. P. Schwan and K. R. Foster, "RF-field interactions with biological systems: electrical properties and biophysical mechanisms," Proceedings of the IEEE, vol. 68, no. 1, pp. 109-113, 1980.

[5] S. S. Chaudhary, R. K. Mishra, A. Swarup, and J. M. Thomas, "Dielectric properties of normal \& malignant human breast tissues at radiowave \& microwave frequencies," Indian Journal of Biochemistry and Biophysics, vol. 21, no. 1, pp. 76-79, 1984.

[6] W. T. Joines, Y. Zhang, C. Li, and R. L. Jirtle, "The measured electrical properties of normal and malignant human tissues from 50 to $900 \mathrm{MHz}$, Medical Physics, vol. 21, no. 4, pp. 547550, 1994.

[7] M. Lazebnik, D. Popovic, L. McCartney et al., "A large-scale study of the ultrawideband microwave dielectric properties of normal, benign and malignant breast tissues obtained from cancer surgeries," Physics in Medicine and Biology, vol. 52, no. 20, pp. 6093-6115, 2007.

[8] S. P. Poplack, T. D. Tosteson, W. A. Wells et al., "Electromagnetic breast imaging: results of a pilot study in women with abnormal mammograms," Radiology, vol. 243, no. 2, pp. 350-359, 2007.

[9] P. M. Meaney, M. W. Fanning, T. Raynolds et al., "Initial clinical experience with microwave breast imaging in women with normal mammography," Academic Radiology, vol. 14, no. 2, pp. 207-218, 2007.

[10] P. M. Meaney, P. A. Kaufman, L. S. Muffly et al., "Microwave imaging for neoadjuvant chemotherapy monitoring: initial clinical experience," Breast Cancer Research, vol. 15, no. 2, article R35, 2013.

[11] N. R. Epstein, A. H. Golnabi, P. M. Meaney, and K. D. Paulsen, "Microwave dielectric contrast imaging in a magnetic resonant environment and the effect of using magnetic resonant spatial information in image reconstruction," in Proceedings of the $33 \mathrm{rd}$
Annual International Conference of the IEEE Engineering in Medicine and Biology Society (EMBS '11), pp. 5738-5741, Boston, Mass, USA, September 2011.

[12] P. M. Meaney, A. H. Golnabi, N. R. Epstein, S. D. Geimer, M. W. Fanning, and K. D. Paulsen, "Integration of a microwave tomographic imaging system with MR for improved breast imaging," Medical Physics, vol. 40, Article ID 103101, 2013.

[13] N. R. Epstein, P. M. Meaney, and K. D. Paulsen, "MR-guided conformal microwave imaging for enhanced inclusion detection within irregularly shaped volumes," in Medical Imaging: Biomedical Applications in Molecular, Structural, and Functional Imaging, Proceedings of SPIE, Lake Buena Vista, Fla, USA, February 2013.

[14] A. H. Golnabi, P. M. Meaney, and K. D. Paulsen, "Tomographic microwave imaging with incorporated prior spatial information," IEEE Transactions on Microwave Theory and Techniques, vol. 61, no. 5, pp. 2129-2136, 2013.

[15] P. M. Meaney, F. Shubitidze, M. W. Fanning, M. Kmiec, N. R. Epstein, and K. D. Paulsen, "Surface wave multipath signals in near-field microwave imaging," International Journal of Biomedical Imaging, vol. 2012, Article ID 697253, 11 pages, 2012.

[16] J. F. Griffith, K. Engelke, and H. K. Genant, "Looking beyond bone mineral density," Annals of the New York Academy of Sciences, vol. 1192, pp. 45-56, 2010.

[17] P. M. Meaney, T. Zhou, D. Goodwin, A. Golnabi, E. A. Attardo, and K. D. Paulsen, "Bone dielectric property variation as a function of mineralization at microwave frequencies," International Journal of Biomedical Imaging, vol. 2012, Article ID 649612, 9 pages, 2012.

[18] P. M. Meaney, D. Goodwin, A. H. Golnabi et al., "Clinical microwave tomographic imaging of the calcaneus: a first-in-human case study of two subjects," IEEE Transactions on Biomedical Engineering, vol. 59, no. 12, pp. 3304-3313, 2012.

[19] N. R. Epstein, P. M. Meaney, and K. D. Paulsen, "3D-parallel microwave tomography for clinical breast imaging," Review of Scientific Instruments. Under review.

[20] D. M. Pozar, Microwave and RF Design of Wireless Systems, John Wiley \& Sons, New York, NY, USA, 2000.

[21] R. M. Sega, "Infrared detection of microwave induced surface currents on flat plates," Final Technical Report Air Force Academy, 1982.

[22] C. A. Balanis, Antenna Theory Analysis and Design, John Wiley \& Sons, New York, NY, USA, 2nd edition, 1997.

[23] A. Taflove and S. C. Hagness, Computational Electrodynamics: The Finite-Difference Time-Domain Method, Atech House, Norwood, Mass, USA, 2nd edition edition, 2000.

[24] C. J. Fox, P. M. Meaney, F. Shubitidze, L. Potwin, and K. D. Paulsen, "Characterization of an implicitly resistively-loaded monopole antenna in lossy media," International Journal of Antennas and Propagation, vol. 2008, Article ID 558078, 9 pages, 2008.

[25] R. M. Irastorza, C. M. Carlevaro, and F. Vericat, "Is there any information on micro-structure in microwave tomography of bone tissue?" Medical Engineering and Physics, vol. 35, no. 8, pp. 1173-1180, 2013.

[26] L. Dun, Microwave imaging spectroscopy for breast cancer diagnosis [thesis], Dartmouth College, 2003.

[27] K. D. Paulsen, P. M. Meaney, M. J. Moskowitz, and J. M. Sullivan Jr., "Dual mesh scheme for finite element based reconstruction algorithms," IEEE Transactions on Medical Imaging, vol. 14, no. 3, pp. 504-514, 1995. 
[28] P. M. Meaney, K. D. Paulsen, B. W. Pogue, and M. I. Miga, "Microwave image reconstruction utilizing log-magnitude and unwrapped phase to improve high-contrast object recovery," IEEE Transactions on Medical Imaging, vol. 20, no. 2, pp. 104116, 2001.

[29] K. D. Paulsen, P. M. Meaney, and L. C. Gilman, Alternative Breast Imaging: Four Model-Based Approaches, Springer, Hanover, NH, USA, 2005.

[30] P. M. Meaney, K. D. Paulsen, and T. P. Ryan, "Two-dimensional hybrid element image reconstruction for TM illumination," IEEE Transactions on Antennas and Propagation, vol. 43, no. 3, pp. 239-247, 1995. 

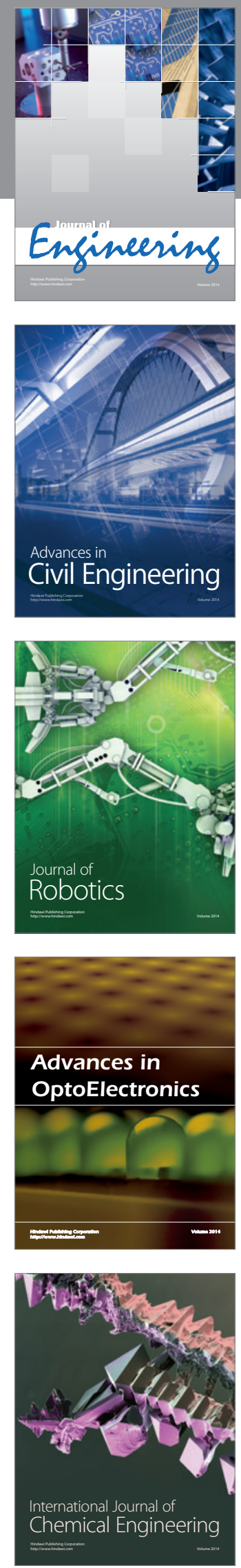

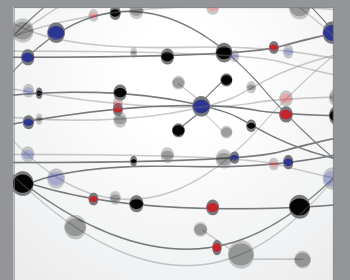

The Scientific World Journal
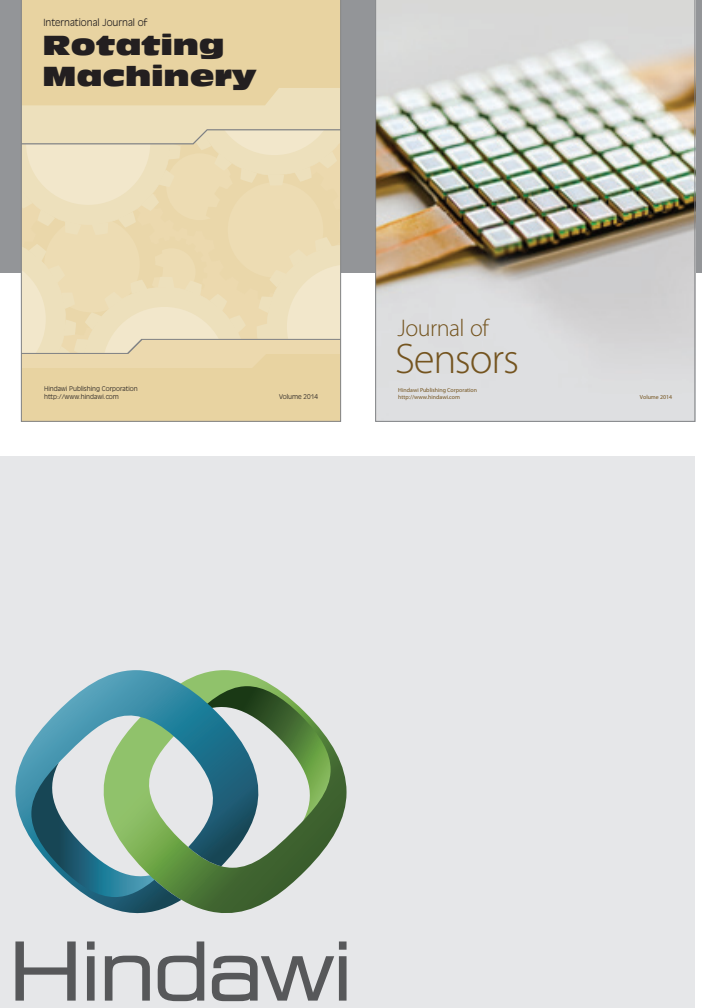

Submit your manuscripts at http://www.hindawi.com
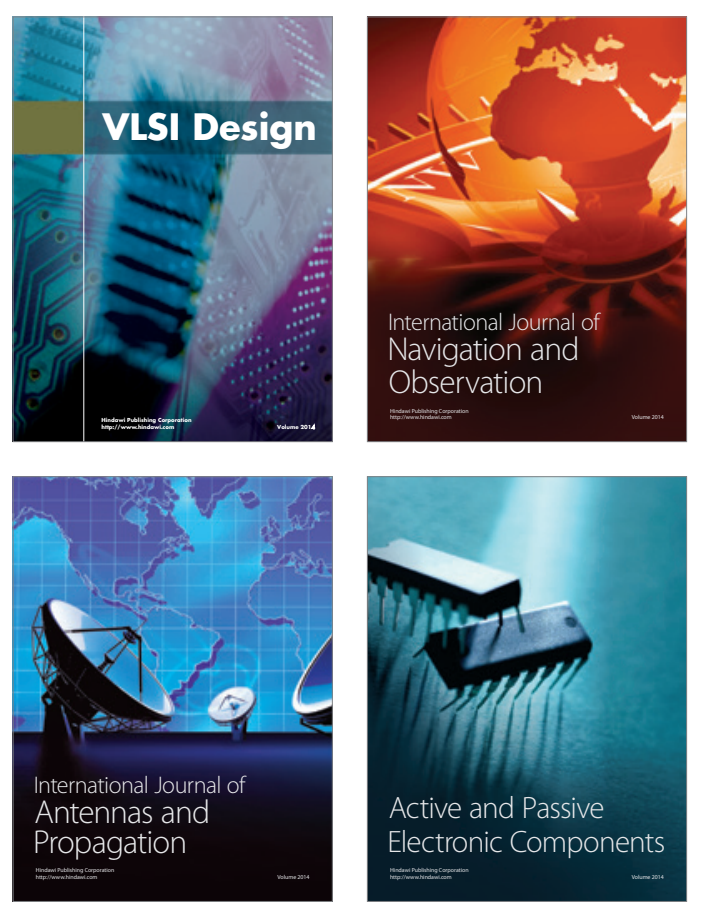
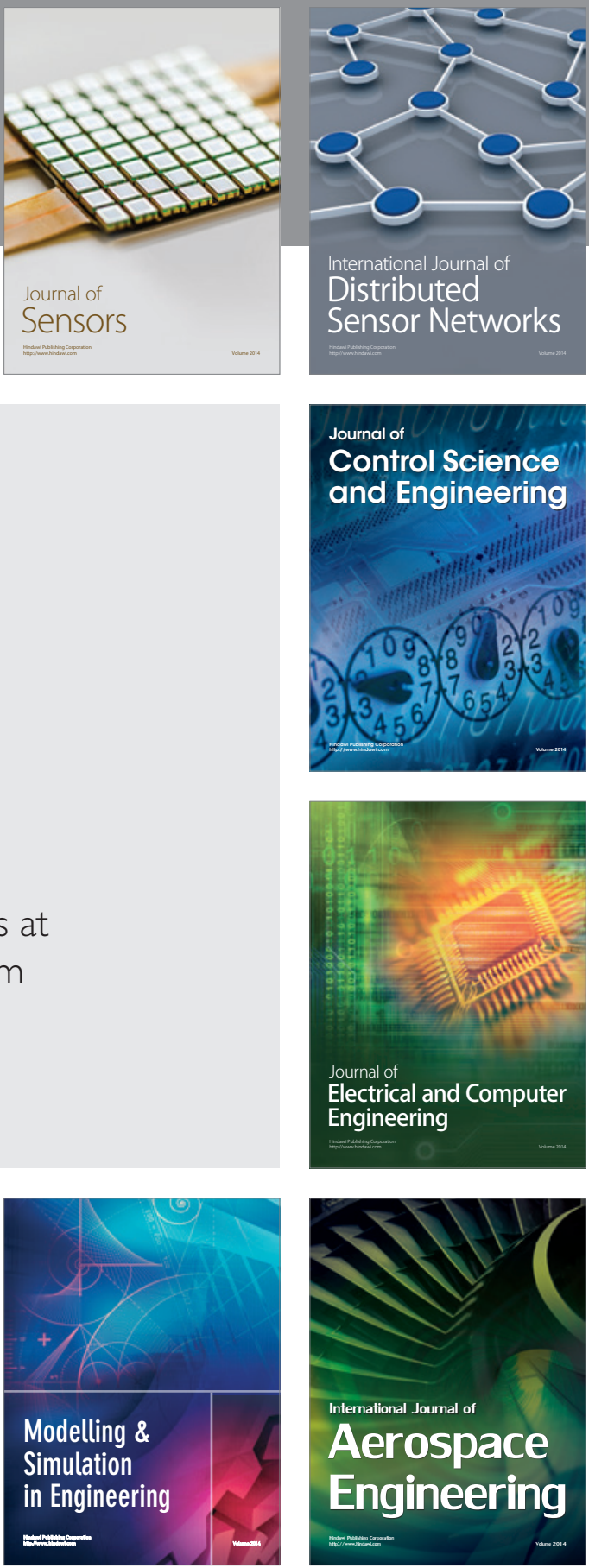

Journal of

Control Science

and Engineering
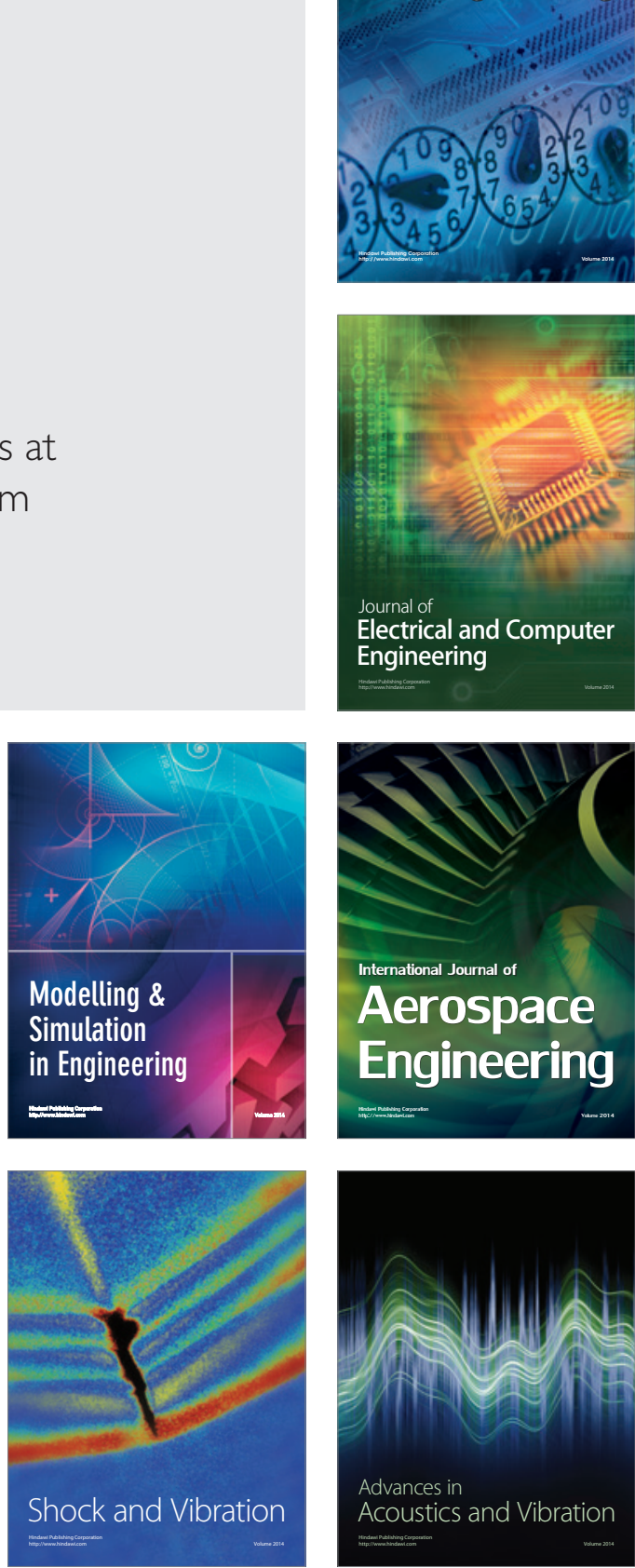